\title{
Editorial for innovative clinical investigations: example of a framework for determining the stages of innovation in surgery or other interventional procedures
}

\author{
Heniz U. Lemke ${ }^{1}$
}

Published online: 11 November 2017

(c) CARS 2017

\section{General comments}

Following the publication of a first set of manuscripts dedicated towards innovative clinical investigations in IJCARS, Vol. 12, May 2017 [1], a considerable number of additional manuscripts have been received which fit into the category of Innovative Clinical Investigations (ICI).

In general, these types of manuscripts focus on how possible new functionalities provided by IT tools and systems are embedded into the practice of medicine, specifically surgery. Within the review process of IJCARS, the authors are encouraged to address questions related to the impact of their innovation on diagnostic/therapeutic workflows, i.e. what is the effective innovation within clinical procedures. The authors should also indicate how these methods/tools potentially impact clinical outcome for the patient, i.e. what is the expected/verified innovation for improved outcomes and their statistical significance.

An appropriate peer-review process has been established in IJCARS, which ensures transparency regarding designation of manuscripts as ICI and corresponding assignment of peer reviewers. It should be noted that reviewers rate the potential importance of a manuscript and its research results and that the ICI designation does not imply an "endorsement" or "award" by IJCARS.

In order to make the importance and fairness of this IJCARS policy on ICI submissions more transparent, a continuing effort is being made to generate a framework/template for the ICI authors to fill out and present it as part of their manuscript submission. This will encourage the authors to give serious thought to where their research fits into the "gen-

\footnotetext{
Heniz U. Lemke

hulemke@cars-int.org

1 Kuessaberg, Germany
}

eral scheme of things", and to present this analysis to the readers of their papers.

There are a number of international initiatives which have made it their objective to assist in the assessment activities of innovative health care technologies, similar to those which are being developed in the context of CARS. These initiatives also try to provide frameworks (e.g. protocol design and validation methods) for assisting investigators and authors in their publication endeavours. Two of the main organizations which fall into this category are: IDEAL Collaboration [2,3] and EXCITE International [4]. EXCITE International, a not-for-profit initiative, is a global collaboration of innovators/industry, regulators, health systems, patients, scientists, end users and other actors in health care, working together to foster health technology innovation and adoption. This approach has been designed to help innovators of breakthrough technologies to achieve accelerated adoption with greater certainty, at a lower cost.

\section{IDEAL Collaboration}

The IDEAL Collaboration is an UK initiative which developed a framework for the stages in innovation in surgery or other interventional procedures defined as idea, development, exploration, assessment and long-term study (IDEAL). The purpose of IDEAL is to improve the quality of research by emphasizing on appropriate methods, transparency of data and rigorous reporting of outcomes.

Applying a formal method such as IDEAL to IJCARS publishing activities, the Development and Exploration stages in IDEAL (see Table 1) seem to provide the right framework classifying manuscripts into the categories of original technical innovation and innovative clinical investigations, respectively. For being publishable in IJCARS, the 8 different study 
Table 1 IDEAL stages (courtesy Peter McCulloch, University of Oxford) and activities marked for IJCARS innovative clinical investigations

\begin{tabular}{|c|c|c|c|c|}
\hline IDEA (Stage 1) & DEVELOPMENT (2A) & EXPLORATION (2B) & ASSESSMENT (3) & LONG TERM STUDY (4) \\
\hline Initial report & $\begin{array}{l}\text { "Tinkering” } \\
\text { (rapid iterative } \\
\text { modification) }\end{array}$ & $\begin{array}{l}\text { Technique now more } \\
\text { stable }\end{array}$ & $\begin{array}{l}\text { Gaining wide } \\
\text { acceptance }\end{array}$ & $\begin{array}{l}\text { Monitoring late and } \\
\text { rare problems, changes } \\
\text { in use \& quality of } \\
\text { surgical performance }\end{array}$ \\
\hline $\begin{array}{l}\text { Innovation may be } \\
\text { planned, accidental } \\
\text { or forced }\end{array}$ & $\begin{array}{l}\text { Small experience } \\
\text { from one centre }\end{array}$ & Replication by others & $\begin{array}{l}\text { Considered as } \\
\text { possible } \\
\text { replacement for } \\
\text { current treatment }\end{array}$ & \\
\hline \multirow[t]{3}{*}{$\begin{array}{l}\text { Focus on } \\
\text { explanation and } \\
\text { description }\end{array}$} & $\begin{array}{l}\text { Focus on technical } \\
\text { details and feasibility }\end{array}$ & $\begin{array}{l}\text { Focks on adverse } \\
\text { effects and potential } \\
\text { benefits }\end{array}$ & $\begin{array}{l}\text { Comparison } \\
\text { against current } \\
\text { best practice (RCT } \\
\text { if possible) }\end{array}$ & \\
\hline & & $\begin{array}{l}\text { Learning curves } \\
\text { important }\end{array}$ & & \\
\hline & & $\begin{array}{l}\text { Definition and } \\
\text { quality parameters } \\
\text { developed }\end{array}$ & & \\
\hline
\end{tabular}

activities suggested in the Development and Exploration stages would need to be carefully selected, defined and appropriately considered in the review process. They may also motivate Clinical Lead Users/Early Adopters to recognize the importance of these stages when engaging in the activities needed for the exploration, assessment and monitoring the dissemination (long-term study) of their R\&D results.

In general, there is a tendency to present publishable results from the activities in the IDEA stage as abstracts in conferences. The CARS Congress may serve here as an example, by providing the possibility to publish long abstracts in the IJCARS Supplement. Publishable activities from the DEVELOPMENT stage typically find their way into regular issues of IJCARS. To be considered for being published in IJCARS as an innovative clinical investigation, manuscripts should have a focus which addresses the EXPLORATION stage, in particular the activities "Focus on adverse effects and potential benefits", "Learning curves important" and "Definition and quality parameters development". Manuscripts which pertain to the IDEAL stages ASSESSMENT and LONG TERM STUDY, typically belong to the domains of dedicated clinical journals and not to IJCARS.

The following provides a brief synopsis and categorization of ten papers in the field of SSEP, CAOS and CAHNS published in this IJCARS issue, in the light of the framework as suggested by IDEAL by specifically fulfilling one or more of the three EXPLORATION stage activities as outlined above.

\section{Surgical simulation, education and planning (SSEP)}

\section{(1) Expert subjective comparison of haptic models for bone-drill interaction}

Thomas Kerwin $^{1}$, Brad Hittle ${ }^{1}$, Sonny Chan ${ }^{2}$, Don Stredney ${ }^{1}$, Gregory Wiet ${ }^{3,4}$

${ }^{1}$ Ohio Supercomputer Center, Columbus, OH, USA ${ }^{2}$ University of Calgary, Calgary, AB, Canada ${ }^{3}$ Department of Otolaryngology, the Ohio State University, Columbus, $\mathrm{OH}$, USA ${ }^{4}$ Nationwide Children's Hospital, Columbus, OH, USA

A spring-damper ball model and a constraint-based model are compared with respect to their effectiveness for improving the expected increase of skill transfer between a simulator and a real surgical environment. A quantitative and qualitative analysis is offered to responses of a survey of 14 otologic surgeons giving their opinion as regards the perceived quality of two haptic algorithms. The validity of this study is based on a paired Wilcoxon signed-rank test for statistically significant observations.

\section{(2) Cost-effective, personalized, 3D-printed liver model for preoperative planning before laparoscopic liver hemihepatectomy for colorectal cancer metastases}

Jan Sylwester Witowski ${ }^{1}$, · Michał Pędziwiatr ${ }^{1}$, Piotr Major1, Andrzej Budzyński ${ }^{1}$ 
1 2nd Department of General Surgery, Jagiellonian University, Medical College, Kraków, Poland

A combination of commercially available software packages is used for creating patient-specific 3D printed liver models at very low cost. The clinical procedure relates to a laparoscopic right hemihepatectomy with the model being made available as a preoperative planning and intraoperative guidance tool. Claims relate to reduce operative time and improve short-term outcomes. Even though the technique can be executed by physicians after a relatively short training period, compared to more expensive methods, the technique as outlined is much slower. Experiences gained, however, relate to only one patient, allowing for no statistical analysis.

\section{(3) Validation of a method for retroperitoneal tumour segmentation}

Cristina Suárez-Mejías ${ }^{1}$, José A. Pérez-Carrasco ${ }^{2}$, Carmen Serrano $^{2}$, José L. López-Guerra ${ }^{3}$, Tomás Gómez-Cía ${ }^{4}$, Carlos L. Parra-Calderón ${ }^{1}$, Begoña Acha ${ }^{2}$

1 Technological Innovation Group, Virgen del Rocío University Hospital, Sevilla ${ }^{2}$ Signal Theory and Communications Department, University of Sevilla ${ }^{3}$ Oncology Unit, Virgen del Rocío University Hospital Sevilla ${ }^{4}$ Surgery Unit, Virgen del Rocío University Hospital, Sevilla, Spain

This paper focuses on a comparison and validation of a proposed new semiautomatic segmentation algorithm with two commercial available segmentation tools. The workflow relates to contouring retroperitoneal tumours on radiotherapy planning workstations or with surgical planning software packages to assist clinical experts for improving accuracy and reducing the amount of time in delineation of tumours. Based on a large selection of metrics, the proposed algorithm allows a faster and more accurate selection of the tumours, avoiding the variability among healthcare professionals in their manual delimitations. The validation is based on 6 experts and no statistical analysis is offered.

(4) Initial experience using a robotic-driven laparoscopic needle holder with ergonomic handle: assessment of surgeons' task performance and ergonomics

Juan A. Sánchez-Margallo ${ }^{1}$, Francisco M. Sánchez-Margallo ${ }^{2}$

1 Department of Computer Systems and Telematics Engineering, University of Extremadura, Badajoz ${ }^{2}$ Jesús Usón Minimally Invasive Surgical Centre, Cáceres, Spain

An assessment is made regarding the adverse effects and potential benefits of robotic-driven needle holder dur- ing suturing tasks in laparoscopic workflows relating, for example, to the restriction of movements due to the fixed position of the surgical ports. Results reported show a similar performance of robotic support with better ergonomics compared to the use of a conventional laparoscopic needle holder during intracorporeal suturing. A relatively small number of six surgeons participated in this study and the statistical analysis is based on the Wilcoxon signed-rank test.

\section{Computer-assisted orthopaedic surgery (CAOS)}

\section{(1) An exploratory study into measuring the cortical bone thickness from $\mathrm{CT}$ in the presence of metal implants}

Tristan Whitmarsh ${ }^{1}$, Graham M. Treece ${ }^{1}$, Andrew H. Gee ${ }^{1}$, Kenneth E. S. Poole ${ }^{2}$

${ }^{1}$ Department of Engineering, University of Cambridge ${ }^{2}$ Department of Medicine, University of Cambridge, UK

The aim of this study was to develop and evaluate metal deletion software for metal artefact removal from postoperative CT scans in 5 different hip repair or replacement procedures: unipolar hemi-arthroplasty, dynamic hip screw fixation, bipolar hemi-arthroplasty, fixation with cannulated screws and total hip arthroplasty. To improve the evaluation of periprosthetic bone after hip replacement surgery may lead to a tool for assessing the progress of fracture healing or to assess the long-term effects of reconstructive surgery and prosthetic replacement surgery. The initial study has focused on 5 patients with the statistical analysis being based on MATLAB version R2013b, Student's T tests and paired T tests.

\section{(2) Error range in proximal femoral osteotomy using computer tomography-based navigation}

Masaki Takao ${ }^{1}$, Takashi Sakai ${ }^{2}$, Hidetoshi Hamada ${ }^{2}$, Nobuhiko Sugano ${ }^{1}$

${ }^{1}$ Department of Orthopaedic Medical Engineering, Osaka University Graduate School of Medicine ${ }^{2}$ Departments of Orthopaedic Surgery, Osaka University Graduate School of Medicine, Japan

The purpose of this study was to determine the error range of a CT-based navigation system employed in procedures such as transtrochanteric rotational osteotomy and curved varus osteotomy. A number of position and angle errors are defined as quality parameters for these procedures. An important observation is that an accurate reproduction of the osteotomy and moved bone fragment position can 
potentially help reduce post-operative discrepancies in leg length. In order to improve surgical accuracy, it would therefore be necessary to develop a computer-assisted device which can track the osteotomized fragments. The validation is based on 7 patients and no statistical analysis is offered.

(3) Validation of three-dimensional models of the distal femur created from surgical navigation point cloud data for intraoperative and post-operative analysis of total knee arthroplasty

David A. J. Wilson ${ }^{1}$, Carolyn Anglin ${ }^{3}$, Felix Ambellan ${ }^{2}$, Carl Martin Grewe ${ }^{2}$, Alexander Tack ${ }^{2}$, Hans Lamecker ${ }^{2,4}$, Michael Dunbar ${ }^{1}$, Stefan Zachow ${ }^{2,4}$

${ }^{1}$ Dalhousie University, Halifax, Nova Scotia, Canada ${ }^{2}$ Zuse Institute Berlin, Berlin, Germany ${ }^{3}$ University of Calgary, Calgary, Alberta, Canada ${ }^{4} 1000$ Shapes GmbH, Berlin, Germany

A statistical shape model (SSM) is intraoperatively acquired in the surgical procedure of navigated total knee arthroplasty and validated with respect to preoperative MRI data. The definition and quality parameters of this study relate to measurement of mismatch between pre- and post-operative femurs retrospectively. They could be integrated into the surgical navigation unit to provide real-time feedback. The validation is based on 10 patients with the statistical analysis being based on pairwise $\mathrm{T}$ tests. If clinical significance is proved through a larger study, the models may be incorporated into a surgical navigation unit, providing a surgeon with accurate real-time feedback and for a given patient, optimization of implant selection and position.

\section{(4) The computer-aided parallel external fixator for complex lower limb deformity correction}

\section{Mengting Wei ${ }^{1}$, Jianwen Chen ${ }^{2,3}$, Yue Guo ${ }^{2,3}$, Hao Sun ${ }^{1}$ \\ ${ }^{1}$ School of Control Science and Engineering, Hebei Uni- versity of Technology, Tianjin ${ }^{2}$ Rehabilitation Hospital, National Research Center for Rehabilitation Technical Aids, Beijing ${ }^{3}$ Key Laboratory of Human Motion Analysis and Rehabilitation Technology of the Ministry of Civil Affairs, Beijing, China}

Using VRML technology, the purpose of this study was to develop and evaluate software to generate an electronic prescription for correction of lower limb deformities. Complex lower limb deformity treated with parallel external fixators and daily strut adjustments can achieve satisfactory outcomes for limb alignment. An important quality parameter is the duration of frame fixation from the time of application to the time of removal. The validation is based on 15 patients and no statistical analysis is offered.

\section{Computer-assisted head and neck surgery (CAHNS)}

\section{(1) Improved surgical procedure using intraoperative navigation for the implantation of the SPG microstimulator in patients with chronic cluster headache}

Carsten Kohlmeier ${ }^{1}$, Peter Behrens ${ }^{2}$, Andreas Böger ${ }^{3}$, Brinda Ramachandran ${ }^{4}$, Anthony Caparso ${ }^{4}$, Dirk Schulze ${ }^{5}$, Philipp Stude $^{6}$, Max Heiland ${ }^{1}$, Alexandre T. Assaf ${ }^{1}$

${ }^{1}$ Department of Oral and Maxillofacial Surgery, University Medical Center Hamburg Eppendorf ${ }^{2}$ Interdisciplinary Pain Center, University Hospital Freiburg, University of Freiburg ${ }^{3}$ Department of Neurology, Pain Clinic, Red Cross Hospital Kassel, Germany ${ }^{4}$ Autonomic Technologies, Inc., Redwood City, CA, USA 5 Digitales Diagnostikzentrum Breisgau, Freiburg ${ }^{6}$ Department of Neurology, University Hospital Bergmannsheil, University of Bochum, Germany

This study investigates intraoperatively navigated patientspecific placement of an SPG microstimulator (typically to be fixed on the posterior maxilla) using custom methods and software for 3D rotatable digitally reconstructed fluoroscopic images, 3D CBCT and a commercially available navigation system. The objective is to match the post-operative CBCT images with the preoperative treatment plan to verify the accuracy of the intraoperative placement of the microstimulator. A significant reduction in the average distance from the SPG microstimulator to the desired target point in patients operated using intraoperative navigation could be observed. The validation is based on 45 patients on whom 24 surgeries were carried out with the new method as compared to the results of 21 patients previously implanted without the assistance of CT navigation. Irradiation duration using intraoperative CBCT was shorter in the navigated group than in the conventional group. The statistical analysis is based on the R Foundation for Statistical Computing, program $\mathrm{R}$ version 3.3.1.

(2) Design, development and clinical validation of computer-aided surgical simulation system for streamlined orthognathic surgical planning

Peng Yuan ${ }^{1}$, Huaming $\mathrm{Mai}^{1}{ }^{1}$ Jianfu $\mathrm{Li}^{1}$, Dennis Chun-Yu $\mathrm{Ho}^{1}$, Yingying Lai ${ }^{1}$, Siting Liu ${ }^{1}$, Daeseung Kim ${ }^{1}$, Zixiang Xiong ${ }^{2}$, David M. Alfi, ${ }^{1,3}$, John F. Teichgraeber ${ }^{4}$, Jaime Gateno $^{1,3,4,5}$, James J. Xia ${ }^{1,3,4,5}$ 
${ }^{1}$ Department of Oral and Maxillofacial Surgery, Houston Methodist Research Institute ${ }^{2}$ Department of Electrical and Computer Engineering, Texas A\&M University ${ }^{3}$ Department of Surgery - Oral and Maxillofacial Surgery, Weill Medical College, Cornell University, New York, NY ${ }^{4}$ Division of Pediatric Plastic Surgery, Department of Pediatric Surgery, the University of Texas Houston Health Science Center ${ }^{5}$ Department of Orthodontics, the University of Texas Houston Health Science Center, Houston, TX, USA

The objective of this study is the discussion of functional modules needed to accurately plan an entire orthognathic surgery within a single software system using computeraided surgical simulation for virtual osteotomy. Positioning of intermediate splints is regarded as the most direct benchmark for measuring the accuracy of the system. 3D cephalometric analysis, including the five geometric properties of orientation, symmetry, position, size and shape, is implemented in a surgical planning system. The validation is based on 10 patients. No statistical analysis is offered although some measures have been taken to prevent conformation bias.

\section{Compliance with ethical standards}

Conflict of interest The author declares that he has no competing interests.

\section{References}

1. Lemke HU (2017) Editorial for clinical investigations: toward the vision and spirit of CARS with innovative clinical investigations. Int J CARS 12(5):789-793. https://doi.org/10.1007/ s11548-017-1586-5

2. McCulloch P, Cook JA, Altman DG, Heneghan C, Diener MK (2013) IDEAL framework for surgical innovation 1: the idea and development stages. BMJ 346. https://doi.org/10.1136/bmj.f3012 (Published 18 June 2013) Cite this as: BMJ 2013;346:f3012

3. Sedrakyan A, Campbell B, Merino JG, Kuntz R, Hirst A, McCulloch P (2016) IDEAL-D: a rational framework for evaluating and regulating the use of medical devices. BMJ 353. https://doi.org/ 10.1136/bmj.i2372 (Published 09 June 2016) Cite this as: BMJ 2016;353:i2372

4. http://www.exciteinternational.com/site/ what-is-excite-international 